\title{
Synchronous Presentation of a Primary Iliac Lymph Node Plasmacytoma and a Prostate Adenocarcinoma
}

\author{
Eiko Klimant ${ }^{\mathrm{a}}$ Daniela M. Albu ${ }^{\mathrm{b}}$ Maurie Markman ${ }^{\mathrm{a}}$ \\ Departments of a Medical Oncology and ${ }^{b} \mathrm{Clinical}$ Research, Cancer Treatment \\ Centers of America, Eastern Regional Medical Center, Philadelphia, Pa., USA
}

\section{Key Words}

Extramedullary plasmacytoma / IL-6 - IL-6 (interferon, beta 2) gene mutation ·

Plasmacytic lymphomas - Primary lymph node plasmacytoma - Prostate adenocarcinoma .

Prostate cancer

\section{Abstract}

Synchronous presentation of primary nodal plasmacytoma and prostate cancer is very rare and has not been described in the literature. Here, we report a case of a patient with nodal plasmacytoma whose clinical presentation was suggestive of metastatic prostate cancer in the setting of recently diagnosed prostate cancer. The workup and treatment of both malignancies as well as a possible underlying common pathologic mechanism (IL-6 gene mutation) are discussed.

\section{Introduction}

Double primary or multiple primary malignancies are mentioned infrequently in the medical literature. Synchronous tumors are primary tumors diagnosed within 6 months of each other. They are presumed to have a common genetic, hormonal, immunologic, environmental or iatrogenic link and are less common than the metachronous tumors (more than 6 months in between diagnosis).

\section{Case Report}

A 68-year-old Caucasian male presented to our institution for further investigation and treatment options of a recently diagnosed stage II prostate adenocarcinoma Gleason score $6(3+3)$, with involvement of the right apex, right lateral mid-region, left base, and left apex, which was consistent 
with bilateral involvement ( 5 out of 12 cores). At presentation, the patient was asymptomatic, with vital signs and weight within normal limits and an unremarkable physical examination.

A CT scan of the abdomen and pelvis performed prior to presentation revealed a left internal iliac chain mass $(5.6 \times 3 \mathrm{~cm})$ of unclear etiology and a nonspecific concentric thickened wall involving the antrum of the stomach, which was further investigated (esophagogastroduodenoscopy with biopsy) and proved to be benign.

The PET/CT scan of the abdomen and pelvis performed at presentation (approximately 1 month from diagnosis) showed a circumscribed ovoid homogeneously enhancing, $4.9 \times 2.7 \mathrm{~cm}$ mass within the left deep pelvis along the iliac chain at the bifurcation of the internal and external iliac artery, which was PET avid (standardized uptake value (SUV) 8.5). Another 5-mm right common iliac chain lymph node demonstrated elevated FDG activity with a maximum SUV of 3.2. Additional small FDG-avid lymph nodes were noted in the common iliac chain and bilateral hila. The hilar lymph nodes measured less than $1 \mathrm{~cm}$ and were non-pathologically enlarged and non-specific, with a maximum SUV of 3.5 on the left and 4.1 on the right side. There was no abnormal FDG activity identified throughout the lung parenchyma bilaterally; additionally, neither axillary lymphadenopathy nor suspicious osseous lesions were detected.

Laboratory results at presentation were as follows: LDH $379 \mathrm{U} / \mathrm{l}$; WBC 8.7; hemoglobin $14.6 \mathrm{~g} / \mathrm{dl}$; hematocrit 42\%; platelets $164 \mathrm{k} / \mu \mathrm{l}$; ANC $4.3 \mathrm{k} / \mu \mathrm{l}$; INR 1, and aPTT $25.3 \mathrm{~s}$. Liver and renal functions were normal. PSA was elevated at $9 \mathrm{ng} / \mathrm{ml}$ at presentation (the PSA level was followed since 1999 when the value was $3.9 \mathrm{ng} / \mathrm{ml}$, showing a gradual increase over a period of 11 years), and the tumor markers CEA, beta-HCG and alpha-fetoprotein were within normal limits. A CT-guided core biopsy of the left iliac chain pelvic mass was performed, showing atypical plasma cells consistent with plasmacytoma. B cell and plasma cell markers CD38, CD138 and lambda light chain were positive.

Further workup for plasmacytoma was completed, revealing the following results: total protein 7.3 $\mathrm{g} / \mathrm{dl}$; protein electrophoresis showed a monoclonal band; IFE showed IgG lambda monoclonal band; total IgG elevated at 1,915 mg/dl (upper normal 1,600 mg/dl); IgA normal at $214 \mathrm{mg} / \mathrm{dl}$; IgM normal at $53 \mathrm{mg} / \mathrm{dl}$; CRP normal at $0.28 \mathrm{mg} / \mathrm{dl}$; sedimentation rate slightly elevated at $17 \mathrm{~mm} / \mathrm{h}$, and beta-2microglobulin $2.84 \mathrm{mg} / \mathrm{l}$ (upper normal $2.51 \mathrm{mg} / \mathrm{l}$ ).

CT-guided bone marrow aspiration and biopsy were performed, showing no evidence of malignancy, with normal cytogenetics and normal karyotype. Also, normal cellular marrow with trilineage maturation was seen. The following elements were in support of the diagnosis of plasmacytoma: monoclonal protein; IgG lambda with an M-spike of $1.2 \mathrm{~g}$; beta-2-microglobulin 2.84 $\mathrm{mg} / \mathrm{dl}$; IgG elevated at $1,915 \mathrm{mg} / \mathrm{dl}$; IgA and IgM in the normal range, and CRP in the normal range. In addition, skeletal survey was negative.

The patient completed a course of stereotactic body radiation therapy to both his prostate and the left iliac chain plasmacytoma. He received a total dose of $36.25 \mathrm{~Gy}$ to his prostate delivered in 5 fractions over a 2-week period of time. Simultaneously, he received radiation treatment to the left iliac plasmacytoma to a dose of $25 \mathrm{~Gy}$, also delivered in 5 fractions. Our patient tolerated the treatments well, with no significant side effects reported.

He presented at the 3-months follow-up feeling well, without significant complaints. However, minimal symptomatology was noted, consisting of intermittent episodes of urinary frequency and urgency, and nocturia (1-2 episodes/night) with no dysuria or hematuria. The patient was therefore continued on Flomax daily.

A restaging CT scan of the chest, abdomen and pelvis, diagnostic with PET, was performed at this visit, which revealed small foci of soft tissue density within the hila bilaterally, not significantly enlarged by CT criteria, with some increased FDG uptake, but essentially stable. The left pelvic mass appeared slightly decreased from prior examination, thus likely representing mild positive response to therapy. The area, which previously measured $5.1 \times 2.6 \mathrm{~cm}$, was now $4.6 \times 2.2 \mathrm{~cm}$. The SUV uptake registered a minimal decrease.

Repeat workup was completed with serum protein electrophoresis, immunofluorescence electrophoresis, beta-2-microglobulin, CRP, erythrocyte sedimentation rate, Ig levels as well as PSA (2.4 $\mathrm{ng} / \mathrm{dl})$. All results were within normal limits, with the exception of the IgG level, which was still above the normal limit but decreased from 1,915 to $1,677 \mathrm{mg} / \mathrm{dl}$. 
At the patient's 6-months follow-up after the initial presentation, the IgG level was stable. In addition, beta-2-microglobulin decreased from 2.9 to $2.78 \mathrm{mg} / \mathrm{l}, \mathrm{M}$-spike was $0.6 \mathrm{~g}$, and PSA $1.7 \mathrm{ng} / \mathrm{dl}$. The imaging studies completed at this visit revealed possible disease progression in the left iliac mass and in the hilar lymph nodes bilaterally. The hilar lymph nodes were biopsied, and the results showed no evidence of malignancy. At the time of publication, the patient's medical management is ongoing.

\section{Discussion}

Prostate cancer is the most common malignancy in American men apart from skin cancer. The medical literature shows synchronous and metachronous presentations of prostate cancer with other cancer types such as renal cell, rectal, breast, bladder testicular and thyroid cancers. Plasma cell dyscrasia may manifest as plasmacytoma, multiple myeloma (MM), primary amyloidosis, or monoclonal gammopathy of unknown significance [1]. Plasmacytomas are subdivided in solitary plasmacytomas of bone and extramedullary plasmacytomas (EMPs).

EMPs constitute $4 \%$ of all plasma cell tumors. They are most commonly (80\%) found in the upper respiratory tract, especially in the nasal cavity and sinuses, nasopharynx, and larynx [2]. However, they may also occur in the gastrointestinal tract, central nervous system, urinary bladder, thyroid, breast, testes, parotid gland, or lymph nodes [2]. Primary lymph node plasmacytomas (PLNPs) are rare malignant neoplasms, which represent $2 \%$ of all EMPs, $0.5 \%$ of lymph node malignant neoplasms, and only $0.08 \%$ of all plasma cell malignant neoplasms [3].

The diagnosis of EMPs is based on the detection of a plasma cell tumor in an extramedullary site and on the absence of MM on bone marrow examination, radiography, and appropriate studies of blood and urine. Their treatment consists of tumoricidal irradiation. The plasmacytoma may recur locally, metastasize to regional nodes, or, rarely, develop into MM [2]. The median age of patients with either solitary bone plasmacytoma or EMP is 55 years [4], which is 10 years younger than that of MM patients $[4,5]$. Less than $7 \%$ of EMP patients will develop a local recurrence after tumoricidal radiation; in one series, all local failures were in bulky tumors $\geq 5 \mathrm{~cm}$ in maximum diameter [6]. Approximately 10-15\% of EMP patients will ultimately develop MM [6]. In the literature, 20 PLNP patients were reported who had a better prognosis than patients with other EMPs, with a low recurrence rate and no progression to myeloma after treatment. Long-term monitoring is mandatory to detect early local recurrence or conversion to MM [7].

On review of genetic profiles of malignancies, a mutation of the IL-6 (interferon, beta 2) gene (chromosomal location $7 \mathrm{p} 21$ ) has been detected in prostate cancer and MM. The CancerIndex/GeneWeb database also lists possible mutations in the IL-6 gene in other cancer types such as breast, renal cell, and ovarian cancers as well as Hodgkin lymphomas and T-cell lymphomas. Additionally, IL-6 is still considered the principal growth factor in the progression of plasma cell disorders $[4,8]$. Although IL-6 is considered a key growth factor for myeloma cells, only a few subpopulations of tumor cells, such as CD45(+) immature cells, proliferate in response to IL-6 [9].

Prostate cancer, while initially dependent on androgens for proliferation, progresses to an androgen-independent state. Evidence has been accumulating that IL- 6 may contribute to prostate cancer progression. Serum levels of IL- 6 correlate with prostate 
tumor burden and patient morbidity. Inhibition of IL-6, with anti-IL-6 antibody, sensitizes androgen-independent prostate cancer cells to chemotherapeutic agents in vitro. This data suggest that targeting IL- 6 may have multiple benefits in prostate cancer patients [10].

IL-6 induces the activation of the androgen receptor in the absence of androgen. IL-6 also modulates vascular endothelial growth factor expression and neuroendocrine differentiation in prostate cells. Anti-IL-6 antibodies showed an inhibitory effect on PC-3 xenografts. Hence, IL-6 is widely considered a promising potential therapeutic target in prostate cancer [9].

This case serves as an excellent example of the critical importance of not simply assuming that observed distant radiographic abnormalities in a patient with a pathologically confirmed localized malignancy (e.g. prostate cancer) actually represent metastatic spread from that primary location. In general, it is essential that the presence of distant metastatic disease be histologically confirmed. In the current case, disease management and overall prognosis were strikingly altered by the additional workup that confirmed the presence of a second malignant condition, rather than this being only a case of widely metastatic prostate cancer.

In summary, our case is the first of synchronous presentation of a PLNP and a prostate adenocarcinoma in the English medical literature. Genetic mutation of the IL-6 gene has been described in several distinct malignancies such as breast cancer, renal cell carcinoma, ovarian cancer, Hodgkin's lymphoma and T-cell lymphoma. Further research on the role of IL-6 in synchronous malignancies as well as on the therapeutic inhibition of IL-6 in synchronous malignancies may be of interest in the future.

\section{References}

$\checkmark 1$ Chim CS, Ooi GC, Loong F, Au AWM, Lie AKW: Side effects and good effects from new chemotherapeutic agents. Case 3. Bortezomib in primary refractory plasmacytoma. J Clin Oncol 2005;23:2426-2428.

2 Rajkumar SV, Kyle RA: Plasma cell disorders; in Goldman L, Ausiello DA (eds): Cecil Medicine. Philadelphia, Saunders Elsevier, 2008, vol 198, p 1434.

-3 Menke DM, Horny HP, Griesser H, Tiemann M, Katzmann JA, Kaiserling E, Parwaresch R, Kyle RA: Primary lymph node plasmacytomas (plasmacytic lymphomas). Am J Clin Pathol 2001;115:119-126.

4 Fanning SR, Hussein MA: Plasmacytoma, extramedullary. http://emedicine.medscape.com/article/207233 (accessed April 7, 2011).

5 Galieni P, Cavo M, Pulsoni A,Awisati G, Bigazzi C, Neri S, Caliceti U, Benni M, Ronconi S, Lauria F: Clinical outcome of extramedullary plasmacytoma. Haematologica 2000;85:47-51.

6 Rajkumar SV: Diagnosis and management of solitary extramedullary plasmacytoma. http://www.uptodate.com/contents/diagnosis-and-management-of-solitary-extramedullary-plasmacytoma (accessed March 31, 2011).

7 Bazaadut S, Soodin D, Singh P, Khalafallah A, Whiters S, Taylor S, Fernando R: Extramedullary plasmacytoma of the tonsil with nodal involvement. Int J Otolaryngol 2010;2010:302656.

-8 Dimopoulos MA, Moulopoulos LA, Maniatis A, Alexanian R: Solitary plasmacytoma of bone and asymptomatic multiple myeloma. Blood 2000;96:2037-2044.

9 Nagel S, MacLeod RAF: IL6 (interleukin 6 (interferon beta 2)). Atlas Genet Cytogenet Oncol Haematol. March 2007. http://atlasgeneticsoncology.org/Genes/IL6ID519ch7p15.html (accessed April 12, 2011).

-10 Smith PC, Hobisch A, Lin DL, Culig Z, Keller ET: Interleukin-6 and prostate cancer progression. Cytokine Growth Factor Rev 2001;12:33-40. 